\title{
ESTADO NUTRICIONAL DE PACIENTES COM INSUFIIIENCIA RENAL CRÔNICA EM HEMODÍ́LISE NO AMAZONAS
}

Rolando Guilhermo Vermehren Valenzuela*, Ângela Gizel Giffoni, Lilian Cuppari, Maria Eugênia Fernandes Canziani

Trabalho realizadona Clínica Renal de Manaus, Manaus, AM

RESUMO - OBjEtivo. Estudar a ingestão alimentar e o estado nutricional de 165 pacientes com IRC em hemodiálise.

Métodos. 0 estado nutricional dos 165 pacientes foi estudado por meio de parâmetros antropométricos, bioquímicos, proteína equivalente do aparecimento do nitrogênio (PNA) e registro do consumo alimentar.

Resultados. Os resultados mostram que a média dos percentuais de adequação do peso, do índice de massa corporal (IMC), da circunferência muscular do braço (CMB) e da prega cutânea do tríceps (PCT) estavam adequados, porém $38 \%$ das mulheres e $27 \%$ dos homens apresentaram PCT abaixo do percentil 5; $39 \%$ dos homens e $2 \%$ das mulheres apresentaram CMB inferiores ao percentil 5, evidenciando uma perda de gordura corporal em ambos os sexos e de massa muscular nos homens. 0 consumo de energia foi de $29,5 \pm 10,2 \mathrm{kcal} / \mathrm{kg} / \mathrm{dia}$, e $74 \%$ dos pacientes consumiam menos que as $35 \mathrm{kcal} / \mathrm{kg} / \mathrm{dia}$ recomendadas para essa população. Apesar do consumo médio de proteína estar dentro do recomendado $(1,32 \pm 0,4 \mathrm{~g} / \mathrm{kg} / \mathrm{dia}), 47 \%$ dos pacientes consumiam menos que $1,2 \mathrm{~g} / \mathrm{kg} / \mathrm{dia}$. A média da albumina sérica foi de $3.96 \pm 0.36 \mathrm{~g} / \mathrm{dL}$ (valor considerado normal). De acordo com as medidas de $\mathrm{PCT}$ e/ou CMB $(\leq 5$ percentil do padrão de referência), $74(44,8 \%)$ pacientes foram classificados como desnutridos.

Conclusão. A desnutrição é freqüente em nossa população e 0 reduzido consumo alimentar pode contribuir para essa condição.

UNITERMOS: Hemodiálise. Avaliação nutricional. Insuficiênciarenal crônica.

\section{INTRODUÇÃO}

Pacientes com insuficiência renal crônica em programa de hemodiálise sofrem, com frequência, de anormalidades nutricionais. Bergstrom etal.' referem que $10 \%$ a $70 \%$ dos pacientes mantidos em hemodiálise e $18 \%$ a $56 \%$ dos pacientes em CAPD apresentam algum sinal de desnutrição. Prevalência semelhante tem sido observada em nosso meio, onde $69 \%$ das mulheres e $79 \%$ dos homens evidenciaram diminuição importante de parâmetros antropométricos ${ }^{2}$.

O impacto da desnutrição sobre a morbidade e mortalidade desses pacientes tem sido amplamente estudado ${ }^{3}$. Vários estudos demonstraram que a baixa adequação de peso e a reduzida concentração sérica de albumina e colesterol aumentam o risco de mortalidade nessa população $3,4,5$.

Diversos fatores podem ser responsáveis pela desnutrição nesses pacientes, entre eles a anorexia com conseqüente redução do consu-

\footnotetext{
*Correspondência: Clínica Renal de Manaus

Av. Castelo Branco, $n^{\circ} 1709$-Cachoeirinha Manaus-AM-CEP 69065-011
}

mo alimentar tem sido apontada como uma das principais causas ${ }^{6,7,8}$. Além disso, outras condições como distúrbios gastrointestinais, acidose metabólica, fatores associados ao procedimento dialítico, distúrbios hormonais, doenças associadas ou intercorrentes (diabetes mellitus, insuficiência cardíaca e infecções) podem também contribuir na fisiopatogênese dessa desnutrição?.

Conhecer e caracterizar adequadamente o estado nutricional de uma população em diálise é fundamental tanto para a prevenção da desnutrição quanto para intervir apropriadamente nos pacientes que já se apresentam desnutridos. Assim, o objetivo do presente trabalho foi caracterizar o estado nutricional de pacientes com insuficiência renal crônica em hemodiálise em um centro de diálise do Amazonas.

\section{Métodos}

Foram estudados 165 pacientes em hemodiálise, na Clínica Renal de Manaus, durante o período de 01/3/1999 a 15/4/1999. Foram incluídos no estudo pacientes maiores de 18 anos de idade, em programa de hemodiálise há pelo menos três meses, e que não apresentavam intercorrências clínicas.
Foram excluídos aqueles em uso de corticosteróides, com infecções recentes ( $<3$ meses), tuberculose em tratamento, doença intestinal, alcoolismo crônico, HIV, doenças malignas, insuficiência cardíaca e pulmonar grave e insucessos de transplante renal nos últimos seis meses.

Os pacientes foram submetidos a uma avaliação nutricional, realizada por nutricionista treinada, que constou de medidas antropométricas, laboratoriais e de consumo alimentar. O registro alimentar de três dias foi a técnica empregada para avaliar a ingestão média de energia, proteína, lipídio, carboidrato, cálcio e fósforo. Os três dias compreendiam um dia em que o paciente se submetia a hemodiálise e dois dias sem o procedimento, excluindo-se sábado e domingo. Além disso, foi analisada a frequiência de consumo dos seguintes grupos alimentares: carnes, laticínios e ovos, cereais e leguminosas e frutas e hortaliças. Os dados coletados da avaliação nutricional foram armazenados e tratados utilizando-se um sistema computadorizado, o "Sistema de apoio à decisão em nutrição - versão 2.5" do Centro de Informática em Saúde da Universidade Federal de São Paulo EPM ${ }^{10}$.

Após a sessão de hemodiálise foram obtidas as seguintes medidas antropométricas: 
Tabela I - Demografia, características clínicas e bioquímicas dos pacientes

\begin{tabular}{|c|c|c|}
\hline \multirow{3}{*}{$\begin{array}{l}\mathrm{N} \\
\text { Sexo }\end{array}$} & \multirow{3}{*}{165} & \multirow[t]{2}{*}{$X \pm D P$} \\
\hline & & \\
\hline & & \\
\hline Masculino & $105(63,6 \%)$ & \\
\hline Feminino & $60(36,4 \%)$ & \\
\hline \multicolumn{3}{|l|}{ Diagnósticos } \\
\hline GNC & $75(45,5 \%)$ & \\
\hline Nefroesc. Hipertensiva & $32(19,4 \%)$ & \\
\hline Diabetes Mellitus & $26(15,8 \%)$ & \\
\hline Obstrutiva & $8(4,8 \%)$ & \\
\hline LES & $5(3,0 \%)$ & \\
\hline Outros & $10(6,0 \%)$ & \\
\hline Indeterminada & $9(5,5 \%)$ & \\
\hline Idade(anos) & & $44,9 \pm 15,0$ \\
\hline Tempo de diálise(meses) & & $33,7 \pm 25,6$ \\
\hline $\mathrm{Kt} / \mathrm{N}$ & & $1,2 \pm 0,4$ \\
\hline Creatinina(mg/dL) & & $11,4 \pm 3,3$ \\
\hline Uréia(mg/dL) & & $|4|, 3 \pm 45,8$ \\
\hline
\end{tabular}

$\mathrm{X} \pm \mathrm{DP}=$ Média e Desvio Padrão; $n=$ Número de Pacientes; $G N C=$ Glomerulonefrite Crônica;

LES = Lúpus EritematosoSistêmico.

Tabela 2 - Parâmetros bioquímicos relacionados ao estado nutricional dos pacientes $(n=165)$

Albumina $(g / d L)$

LinfócitosTotais $\left(\mathrm{mm}^{3}\right)$

Colesterol $(\mathrm{mg} / \mathrm{dL})^{*}$

Triglicerídeo $(\mathrm{mg} / \mathrm{dL})^{*}$

Transferrina $(\mathrm{mg} / \mathrm{dL})$

Hematócrito(\%)

Hemoglobina(g)

Fósforo(mg/dL)

Cálcio $(\mathrm{mg} / \mathrm{dL})$

$\mathrm{PTH}(\mathrm{pg} / \mathrm{mL})$

\begin{tabular}{c}
$\mathbf{X} \pm \mathbf{D P}$ \\
\hline $4,0 \pm 0.4$ \\
$1295.0 \pm 509,3$ \\
$149,7 \pm 44,7$ \\
$165,0 \pm 87,6$ \\
$148,7 \pm 59.8$ \\
$32,7 \pm 5.4$ \\
$10,9 \pm 1,8$ \\
$5,6 \pm 1,9$ \\
$8,9 \pm 1,4$ \\
$238,8 \pm 217,1$
\end{tabular}

$X \pm D P=$ Média e Desvio Padrão; $n=$ Número de Pacientes; $P T H=$ Paratormônio; ${ }^{*} n=\mid 49$ pacientes.

peso corporal, estatura, prega cutânea trícipital (PCT), bícipital, subescapular e suprailíaca (utilizando-se o adipômetro Lange) e a circunferência do braço $(C B)$. Os seguintes parâmetros foram calculados: peso ideal para estatura e compleição física " e índice de massa corporal (IMC), calculado pela razão entre o peso e o quadrado da estatura. A seguinte fórmula foi empregada no cálculo da circunferência muscular do braço $(\mathrm{CMB}): \mathrm{CMB}(\mathrm{cm})$ $=\mathrm{CB}(\mathrm{cm})-(\mathrm{PCT} \text { em } \mathrm{mm} \times 0,3 / 4)^{12} \cdot \mathrm{E}$ a estimativa da porcentagem de gordura corporal foi realizada utilizando a seguinte formula: $\%$ de gordura $=4,95 /$ densidade corporal - 4,50 $\times 100^{13}$, sendo considerados níveis normais de 15 a $18 \%$ para homens e 20 a $25 \%$ para mulheres ${ }^{14}$. A adequação da PCT e da CMB foi calculada, utilizando-se a distribuição de Frisancho $0^{12}$ em relação aos $5^{\circ}, 10^{\circ}$, $25^{\circ}, 50^{\circ}, 75^{\circ}, 90^{\circ}$ e $95^{\circ}$ percentis definidos para a população normal. A classificação do estado nutricional baseou-se nas medidas da PCT e CMB, considerando-se como desnutrido ou com risco de desnutrição os pacientes com valores de PCT e/ou CMB menor ou igual ao percentil 5 do padrão de referência ${ }^{12}$. Vale ressaltar as dificuldades em se caracterizar desnutrição na população de pacientes com IRC. Os padrões de referência das medidas antropométricas não são específicos para essa população, e nem mesmo para a população saudável brasileira, entretanto, essa ainda é a forma de avaliação nutricional mais utilizada e era a empregada na rotina da nutricionista da clínica. Além disso, as variações da água corporal podem influenciar as medidas antropométricas.

Foram analisados ainda parâmetros bioquímicos: uréia, creatinina, colesterol total e triglicerídeos, transferrina, cálcio e fósforo. A albumina foi medida pelo método verde de bromocresol (valor normal de 3,5 a 4,8g/dL)e - PTH pela técnica imunoquimioluminométrica (valor normal de $10 \mathrm{a} 65 \mathrm{pg} / \mathrm{mL}$ ). Entre os parâmetros hematimétricos foram determinados hemoglobina, hematócrito e leucócitos totais. Aingestão protéicafoi estimada calculando-se o equivalente protéico do aparecimento do nitrogênio(PNA) $)^{15}$. A eficiência da diálise foi estimada por meio do cálculo do Kt/ $\mathrm{V}$ pela fórmula de Daugirdas II'.

As variáveis categóricas serão apresentadas como freqüências e percentuais e as variáveis contínuas como média e desvio padrão. $O$ teste do Qui-quadrado foi empregado para testar a associação entre as variáveis categóricas e $o$ teste $t$ de Student para comparação entre médias dos grupos. $O$ coeficiente de correlação de Pearson foi utilizado para analisar associações entre os parâmetros antropométricos, bioquímicos, hematológicos e PNA. Fixou-se nível de significância de $5 \%(a=0,05)$ para todos os testes. Os programas utilizados na análise estatística foram: Epi-Info (versão: 6.04b) e EXCEL 97.

\section{Resultados}

Os dados demográficos eas características clínicas dos 165 pacientes são encontrados na Tabela I. Como podemos observar, $64 \%$ dos pacientes eram do sexo masculino com idade variando de 18 a 84 anos. 0 tempo de tratamento dialítico variou de 3 a 127 meses, e a principal etiologia da insuficiência renal foi glomerulonefrite crônica $45,5 \%$. Observamos, ainda, que a média do $\mathrm{Kt} / \mathrm{V}$ da diálise foi de I,2, entretanto o Kt/ $\mathrm{V}$ foi inferior a esse valor em $53 \%$ dos pacientes.

Como podemos observar na Tabela 2 , as médias dos níveis séricos de albumina, colesterol e transferrina encontravam-se dentro dos limites da normalidade. Somente $8 \%$ dos pacientes apresentavam níveis de albumina abaixo de $3,5 \mathrm{~g} / \mathrm{dL}$. Observamos ainda, que a média dos linfócitos se encontrava abaixo do normal, sendo que em $74 \%$ dos pacientes esta contagem era inferior a $1.500 / \mathrm{mm}^{3}$. As concentrações de cálcio, fósforo e PTH encontra- 
Figura I - Distribuição dos pacientes quanto ao consumo das principais fontes alimentares em pelo menos um dos três dias avaliados $(n=165)$
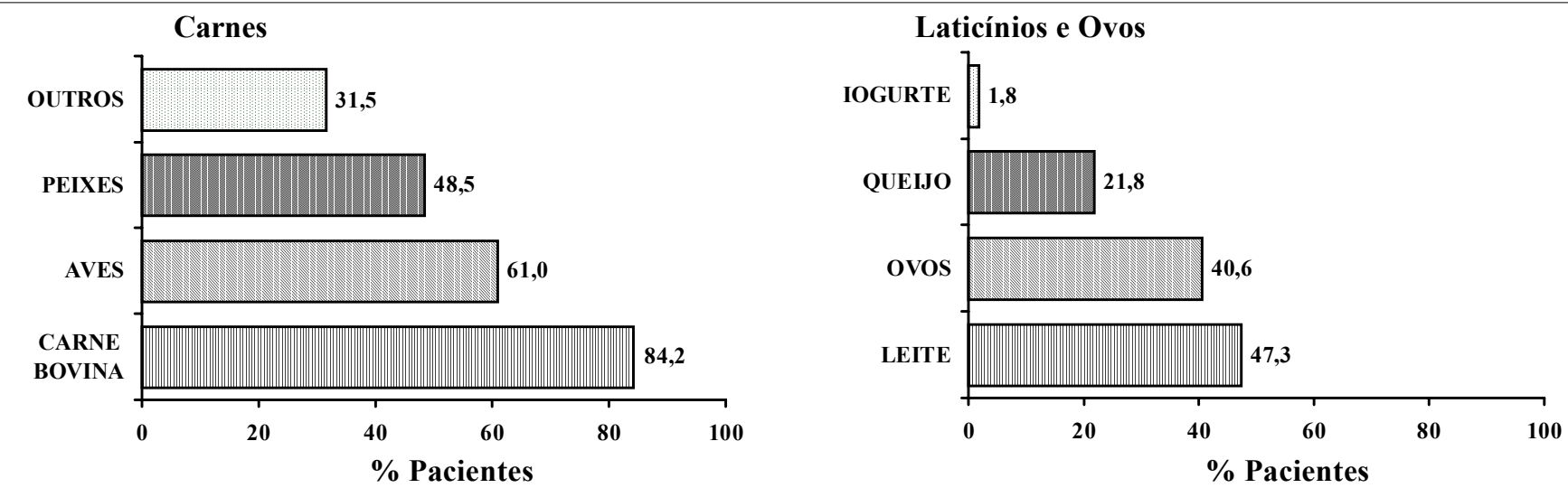

Cereais e Leguminosas

Frutas e Hortaliças
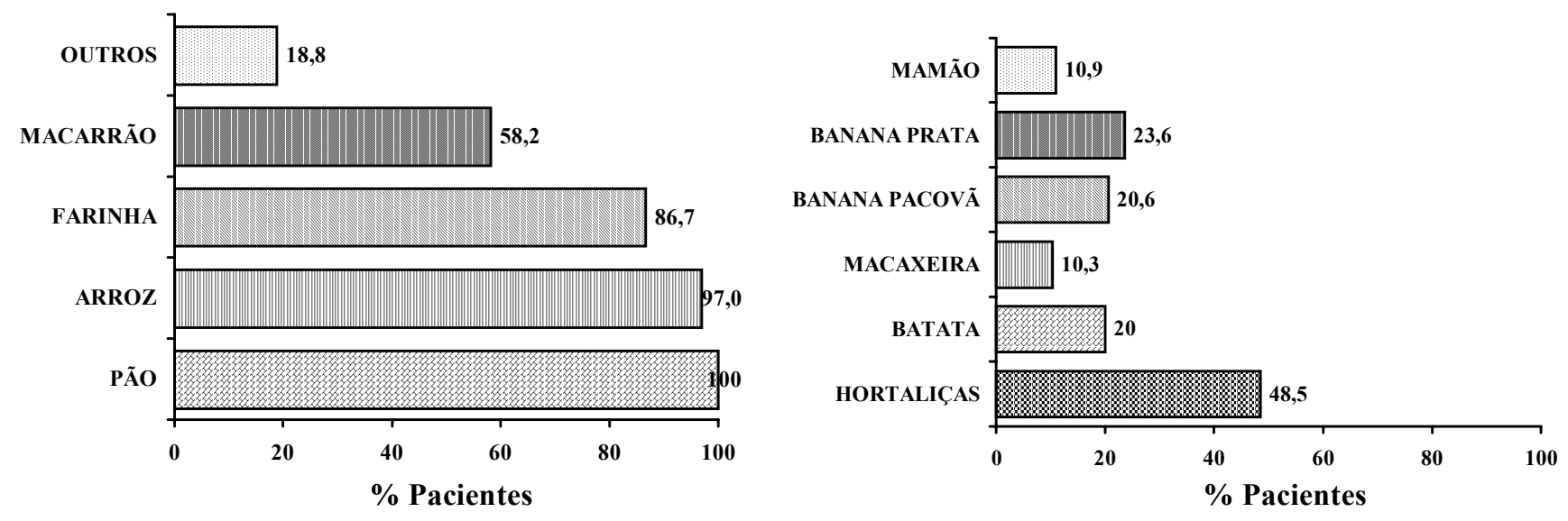

Tabela 3 - Consumo alimentar e proteína equivalente do aparecimento do nitrogênio (PNA) dos pacientes $(n=165)$

\begin{tabular}{lc} 
& $\mathbf{X} \pm \mathbf{D P}$ \\
\cline { 2 - 2 } Energia (kcal/kg/dia) & $29,5 \pm 10,2$ \\
Proténa (\%VCT) & $18,5 \pm 3,7$ \\
Carboidrato(\%VCT) & $52,0 \pm 8,5$ \\
Lipídios (\%VCT) & $29,5 \pm 7,2$ \\
Proténas (g/Kg/dia) & $1,32 \pm 0,4$ \\
PAVB (\%) & $61,7 \pm 13,3$ \\
PNA (g/kg/dia) & $1,1 \pm 0,4$ \\
Cálcio (mg/dia) & $307, I \pm 154,5$ \\
Fósforo (mg/dia) & $774,7 \pm 278,7$
\end{tabular}

$\mathrm{X} \pm \mathrm{DP}=$ Média e Desvio Padrão; $n$ = Número de Pacientes; Kcal = Quilocalorias;

VCT = Valor Calórico Total; $P A V B=$ Proténas deAlto Valor Biológico;

PNA = Proténa EquivalentedoAparecimento do Nitrogênio.

vam-se em média dentro do esperado para pacientes em hemodiálise.

Os resultados do consumo alimentar avaliado pelos registros alimentares encontramse na Tabela 3. O consumo de energia foi de
$29,5 \pm 10,2 \mathrm{kcal} / \mathrm{kg} / \mathrm{dia}$, sendo que em $61 \%$ dos pacientes esteve abaixo de $35 \mathrm{kcal} / \mathrm{kg} / \mathrm{dia}$. O consumo protéico foi de I,32 $\pm 0,4 \mathrm{~g} / \mathrm{kg} /$ dia, entretanto em $47 \%$ dos pacientes era inferior a I, $2 \mathrm{~g} / \mathrm{kg} /$ dia e em $24 \%$ abaixo de I,0 $\mathrm{g} / \mathrm{kg} / \mathrm{dia}$. Observou-se ainda que, em média, $62 \%$ das proteínas consumidas eram de alto valor biológico. A estimativa de ingestão protéica avaliada pelo cálculo do PNA foi I, I 4 $\pm 0,35 \mathrm{~g} / \mathrm{kg} / \mathrm{dia}$ e em $42 \%$ dos pacientes os valores encontravam-se abaixo de $\mathrm{lg} / \mathrm{kg} / \mathrm{dia}$.

Verificou-se, em média, um reduzido consumo de cálcio, com $89 \%$ dos pacientes consumindo menos que $500 \mathrm{mg} / \mathrm{dia}$. Em relação ao fósforo, o consumo médio esteve dentro dos limites recomendados para pacientes renais crônicos.

A freqüência de consumo dos principais grupos alimentares encontra-se na Figura I. Como podemos observar, carne bovina, leite, pão, arroz, farinha e hortaliças foram consumidos em pelo menos um dos três dias avaliados pela maioria dos pacientes nos diferentes grupos alimentares.

Os dados antropométricos estão apresentados na Tabela 4. A média do IMC esteve dentro do limite de normalidade $\left(18,5\right.$ a $\left.24,9 \mathrm{Kg} / \mathrm{m}^{2}\right)$ 


\begin{tabular}{|c|c|c|c|}
\hline & Total $(n=165)$ & Feminino $(n=60)$ & Masculino $(n=105)$ \\
\hline Altura $(\mathrm{cm})$ & $158,6 \pm 8,6$ & $150,9 \pm 5,7$ & $163,1 \pm 6,6 *$ \\
\hline PesoAtual (kg) & $58,8 \pm 10,7$ & $52,6 \pm 9,7$ & $62,4 \pm 9,5 *$ \\
\hline Adeq. do Peso(\%) & $103,5 \pm 13,4$ & $101,9 \pm 14,8$ & $104,4 \pm 12,5$ \\
\hline IMC $\left(\mathrm{kg} / \mathrm{m}^{2}\right)$ & $23,3 \pm 3,4$ & $23, I \pm 3,8$ & $23,4 \pm 3,1$ \\
\hline Adeq. da CMB (\%) & $92,8 \pm 10,3$ & $99,9 \pm 9,2$ & $88,7 \pm 8,6 *$ \\
\hline Adeq.daPCT(\%) & $67,8 \pm 38,5$ & $68,0 \pm 35,0$ & $67,6 \pm 40,5$ \\
\hline \%Gord.Corporal & $21,1 \pm 9,4$ & $28,9 \pm 8,0$ & $16,6 \pm 7,0 *$ \\
\hline
\end{tabular}

$\mathrm{n}=$ Número de Pacientes; $\mid \mathrm{MC}=$ Índice de Massa Corporal; $\mathrm{CMB}=$ Circunferência Muscular do Braço; $\mathrm{PCT}=$ Prega Cutânea Tricipital; ${ }^{*} p<0,000$ I femininovs masculino.

Tabela 5 - Demografia, características clínicas e bioquímicas dos grupos desnutrido (PCT e/ou CMB $\leq$ percentil 5) e não desnutrido (PCT e/ou CMB > percentil 5)

\begin{tabular}{|c|c|c|}
\hline & Desnutridos $(n=74)$ & Não Desnutridos (n=9l) \\
\hline Sexo $M / F$ & $51 / 23$ & $54 / 37$ \\
\hline Idade(anos) & $45,4 \pm 15,4$ & $44,5 \pm 14,6$ \\
\hline Tempodediálise(meses) & $36,9 \pm 27,7$ & $29,9 \pm 22,3$ \\
\hline $\mathrm{Kt} N \mathrm{~N}$ & $1,2 \pm 0,2$ & $1,3 \pm 0,4$ \\
\hline Creatinina(mg/dL) & $11,4 \pm 3,0$ & $11,4 \pm 3,6$ \\
\hline Uréia(mg/dL) & $129,5 \pm 40,9$ & $150,9 \pm 47,4^{*}$ \\
\hline Albumina(g/dL) & $3,9 \pm 0,4$ & $4,0 \pm 0,4$ \\
\hline Linfócitos Totais $\left(\mathrm{mm}^{3}\right)$ & $|27|, 2 \pm 409,8$ & $|3| 4,2 \pm 579,4$ \\
\hline Colesterol (mg/dL) & $152,3 \pm 50,9$ & $147,7 \pm 39,0$ \\
\hline Triglicerídeo(mg/dL) & $159,8 \pm 78,9$ & $169,3 \pm 94,3$ \\
\hline Transferrina(mg/dL) & $140,7 \pm 60,8$ & $155,2 \pm 58,5$ \\
\hline Fósforo $(\mathrm{mg} / \mathrm{dL})$ & $5,2 \pm 1,8$ & $5,8 \pm 1,9 *$ \\
\hline Cálcio(mg/dL) & $8,9 \pm 1,7$ & $8,8 \pm 1,1$ \\
\hline PTH (pg/mL) & $236,5 \pm 226,1$ & $240,8 \pm 210,8$ \\
\hline
\end{tabular}

$\mathrm{n}=$ Número de Pacientes; $\mathrm{CMB}=$ Circunferência Muscular do Braço; $\mathrm{PCT}=$ Prega Cutânea Tricipital; ${ }^{*} \mathrm{p}<0,05$ desnutridos vs não desnutridos; $\mathrm{M}=$ Masculino; $\mathrm{F}=$ Feminino; $\mathrm{PTH}=$ Paratormônio.

e não diferiu entre os sexos. Entretanto, em $25 \%$ dos pacientes esse índice estava acima de $25 \mathrm{~kg} / \mathrm{m}^{2}$ e em $4 \%$ abaixo de $18,5 \mathrm{~kg} / \mathrm{m}^{2}$. Na população como um todo, a adequação da $\mathrm{CMB}$, em média, esteve dentro do normal, de 90 a $110 \%$, entretanto no sexo masculino a adequação foi significativamente menor que no sexo feminino e encontrava-se abaixo da faixa de eutrofia. Já a adequação da PCT encontrava-se abaixo da normalidade $(60 \%$ a $1 \mathrm{l} 0 \%)$ em ambos os sexos e a porcentagem de gordura corporal esteve dentro da normalidade no sexo masculino e se apresentava aumentada no sexo feminino.

Não houve correlação significante entre a PCT ou CMB coma idade, eficiência da diálise, etiologia da insuficiência renal, renda familiare escolaridade.

$\mathrm{Na}$ Tabela 5 apresentamos a comparação entre os pacientes desnutridos ( $44,8 \%)$ e não desnutridos (55,2\%). Não observamos diferenças quanto ao sexo, idade, etiologia da insuficiência renal crônica, tempo em diálise, $\mathrm{Kt} / \mathrm{V}$ e os níveis séricos de creatinina. Entretanto, os níveis de uréia e de fósforo dos pacientes desnutridos foram significativamente menores.

O consumo de energia foi semelhante nos dois grupos $(29,16 \pm 10,13$ vs. $29,77 \pm 0,34$ $\mathrm{kcal} / \mathrm{kg} / \mathrm{dia}$ ), entretanto observamos que 0 percentual de energia proveniente de carboidratos era significantemente mais elevado e 0 de lipídios mais baixo nos pacientes desnutridos $(52,8 \pm 8, I$ vs. $50,6 \pm 8,5 \%, p=0,01$ e $28, I \pm 7$, I vs. $30,6 \pm 7,0 \%, p=0,02$; respectivamente). OPNA foi significativamente menor nos pacientes desnutridos $(I, 08 \pm 0,32 \mathrm{vs}$. $1,19 \pm 0,37 \mathrm{~g} / \mathrm{kg} / \mathrm{dia}, \mathrm{p}=0,03)$. Não observamos diferença significante em relação à renda familiar e escolaridade entre os dois grupos.

\section{Discussão e conclusões}

Este estudo teve como objetivo caracterizar o estado nutricional de pacientes em programa de hemodiálise no Amazonas, onde existe uma população de etnias, culturas e bases alimentares diferenciadas.

Nossos resultados confirmam a elevada prevalência de desnutrição energético-protéica também em nossa população. Dos 165 pacientes estudados, $45 \%$ apresentavam desnutrição identificada por prega cutânea triciptal e/ou circunferência muscular do braço menor ou igual ao percentil 5 do padrão de referência da população dos Estados Unidos. Outros autores ${ }^{2}$ também consideram que medidas antropométricas abaixo do $5^{\circ}$ percentil indicam alto risco ou presença de desnutrição energético-protéicagrave.

Vários trabalhos na literatura demonstram uma ampla variação na prevalência de desnutrição em pacientes hemodialisados. Na maioria desses relatos, a prevalência varia de $10 \%$ a $70 \%$ '. Esta ampla variação deve-se, provavelmente, às diferenças nas populações estudadas e aos critérios utilizados na avaliação do estado nutricional.

Em nossa população, as médias de adequação do peso e IMC estavam dentro da faixa de normalidade. Amédia do peso corporal dos pacientes estudados $(63 \mathrm{~kg})$ foi semelhante ao dos indivíduos saudáveis das Regiões Norte, Nordeste e Sul do Brasil $(60,3 \mathrm{~kg}, 57,5 \mathrm{~kg}$ e $63,7 \mathrm{~kg}$, respectivamente) ${ }^{17}$. A média do IMC dos pacientes $\left(23 \mathrm{~kg} / \mathrm{m}^{2}\right)$ também não foi menor que o IMC dos indivíduos saudáveis dessas Regiões $\left(22 \mathrm{~kg} / \mathrm{m}^{2}, 21 \mathrm{~kg} / \mathrm{m}^{2}\right.$ e $22 \mathrm{~kg} / \mathrm{m}^{2}$, respectivamente). A falta de repercussão da desnutrição sobre o peso corporal e o IMC poderia ser parcialmente explicada pela hiperhidratação desses pacientes, que aumenta 0 peso corporal com pouca repercussão nas medidas da PCT e da CMB. Em nosso estudo, a CMB esteve abaixo da faixa de eutrofia somente nos pacientes do sexo masculino, demonstrando maior perda de massa muscular nos homens. Já a adequação da PCT esteve baixa em ambos os sexos sem diferença entre eles. Cuppari et al. ${ }^{2}$, em nosso meio, também relataram diminuição de massa muscular mais importante no sexo masculino, entretanto, diferindo dos nossos resultados, observaram uma maior redução de gordura no sexo feminino. Por outro lado, a porcentagem de gordu- 
ra corporal dos homens encontrava-se adequado e aumentado nas mulheres, utilizandose a referência de $15 \%$ a $18 \%$ para homens e de $20 \%$ a $25 \%$ para mulheres. A discordância entre os resultados de adequação da PCT e porcentagem de gordura nas mulheres pode ser parcialmente explicado pela diferença nas populações de referência utilizadas.

Muitos fatores de risco para ocorrência de desnutrição em pacientes dialisados têm sido estudados. Lowrie et al. ${ }^{5}$ observaram um risco maior de desnutrição em pacientes diabéticos, enquanto Movill et al. ${ }^{18}$ observaram uma maior prevalência de desnutrição em pacientes idosos, principalmente com idade superior a 75 anos. Em nosso estudo, não observamos correlação entre a etiologia da IRC e a idade com as medidas antropométricas. O tempo em diálise parece também influenciar negativamente no peso e na composição corporal ${ }^{19}$. Em nosso estudo, não observamos diferença entre tempo de diálise e os parâmetros antropométricos, ao compararmos os grupos de desnutridos e não desnutridos. Talvez 0 fato de nossa população ser constituída de pacientes jovens, em diálise por curto período de tempo e com poucos diabéticos, possa explicar de não termos observado essas associações.

Vários estudos demonstraram correlação entre estado nutricional e eficiência de diálise, medida pelo $\mathrm{Kt} / \mathrm{V}^{20}$. No presente trabalho, não observamos correlação entre $\mathrm{Kt} / \mathrm{V}$ e parâmetros antropométricos, entretanto $52 \%$ dos pacientes estavam com Kt/V abaixo do recomendado de $1.2 \mathrm{e}$, ao compararmos os grupos desnutridos e não desnutridos, o Kt/V era menor no grupo desnutrido, porém essa diferença atingiu o limite de significância estatística $(p=0,059)$.

A maioria das médias dos parâmetros bioquímicos gerais encontrava-se dentro do esperado para pacientes em hemodiálise crônica. Entretanto, em $38 \%$ e $32 \%$ dos pacientes, a uréia e a creatinina, respectivamente, apresentaram valores considerados baixos. Lowrie et al. ${ }^{21}$ descreveram que pacientes com níveis de uréia e creatinina reduzidos apresentavam maior risco de morte. Estes autores sugerem que esses níveis reduzidos estavam associados com baixo consumo de proteínas e diminuição da massa muscular esquelética. Em nosso estudo, tanto a uréia sérica quanto o PNA foram significativamente menores no grupo de pacientes desnutridos, sugerindo um menor consumo de proteínas, todavia observamos que o consumo de proteínas avaliado pelo registro alimentar não foi diferente entre desnutrido e não desnutrido, o que pode ser parcialmente justificado pela baixa precisão da técnica de consumo alimentar empregada ${ }^{22}$.

As médias de albumina, colesterol e transferrina estavam dentro dos limites de normalidade nos pacientes estudados. Não encontramos correlação entre os níveis de albumina e os parâmetros antropométricos, e apenas $8 \%$ dos pacientes apresentam albumina menor que $3,5 \mathrm{~g} / \mathrm{dL}$. Além disso, não houve diferença nos níveis de albumina entre desnutridos e não desnutridos. No presente estudo, a albumina não se comportou como um marcador de desnutrição. Outros autores também observaram a baixa sensibilidade da albumina para detectar desnutrição ${ }^{8}$.

Alguns estudos sugerem uma associação entre PTH e desnutrição. Em nosso estudo, não observamos diferença nos níveis de PTH entre os grupos desnutridos e não desnutridos. Além disso, não encontramos correlação entre PTH e parâmetros antropométricos. O PTH pode estar relacionado com desnutrição, quando relativamente baixo ou quando muito elevado. A desnutrição associada a níveis baixos de PTH parece ser um reflexo do baixo consumo alimentar, enquanto que o PTH, quando muito elevado, possivelmente por sua ação catabólica, também pode contribuir indiretamente para 0 desenvolvimento ou piora da desnutrição. Na população estudada encontramos elevada freqüência de PTH abaixo de $200 \mathrm{pg} / \mathrm{mL}$, que provavelmente está mais associada a outros fatores relacionados à osteodistrofia renal e não à baixa ingestão alimentar.

A contagem total de linfócitos também pode ser utilizada como um indicador do estado nutricional ${ }^{23} \mathrm{e}$ valores abaixo de $1.500 \mathrm{~mm}^{3}$ sugerem desnutrição energético-protéica ${ }^{24}$. Marcen et al..$^{25}$ encontraram uma associação entre desnutrição e linfopenia em pacientes submetidos à hemodiálise. Alguns fatores, entre eles, deficiência de zinco ${ }^{26}$ podem causar linfopenia em pacientes renais crônicos. $O$ fato de $74 \%$ dos pacientes estudados apresenta- rem contagem de linfócitos totais abaixo de $1.500 \mathrm{~mm}^{3}$ não permite a utilização desse parâmetro como marcador nutricional em nossa população.

Além dos fatores catabólicos inerentes à insuficiência renal crônica e aos métodos dialíticos ${ }^{9}$, a presença de desnutrição energético-protéica em pacientes dialisados está comumente associada ao baixo consumo alimentar, principalmente de energia ${ }^{6}$. Conforme já comentado, a ingestão protéica aproxima-se do recomendado para manutenção do balanço nitrogenado; por outro lado, o consumo de energia dos pacientes aqui estudados esteve em média $5,5 \mathrm{kcal} / \mathrm{kg} / \mathrm{dia}$ ( $16 \%$ ), abaixo do recomendado para pacientes em hemodiálise. Vale ressaltar que em apenas $26 \%$ dos pacientes o consumo de energia estava dentro do recomendado. Esses dados estão de acordo com estudos de outros países $^{7}$ e em nosso meio ${ }^{2}$.

Apesar do consumo energético insuficiente dos nossos pacientes, a análise da distribuição do consumo de energia mostrou que os percentuais provenientes de carboidratos, proteínas e lipídios não diferem daquela proposta para indivíduos normais, fazendo supor que o déficit alimentar seja global.

$O$ fato de $61 \%$ dos pacientes apresentarem um consumo de energia reduzido $(<30$ $\mathrm{kcal} / \mathrm{kg} / \mathrm{dia}$ ) e $41 \%$ consumo baixo de proteínas $(<1.2 \mathrm{~g} / \mathrm{kg} / \mathrm{dia})$ sugere que esses pacientes possam estar em balanço nitrogenado negativo, sendo este fator importante na determinação do estado nutricional dessa população.

Nos pacientes estudados, o consumo médio protéico avaliado pelo registro alimentar foi de I,3 g/ $/ \mathrm{kg} / \mathrm{dia}$, valor esse considerado seguro para manter o balanço nitrogenado neutro ou positivo ${ }^{22}$. Em estudos nacionais ${ }^{17,27}$ de populações saudáveis, observa-se que a ingestão de proteínas fica ao redor de $100 \mathrm{~g} /$ dia em cidades das Regiões Sul e Sudeste. Já na cidade de Belém do Pará, esse consumo é de $77 \mathrm{~g} / \mathrm{dia}$, semelhante ao observado no presente estudo (75 g/dia). Isso sugere, portanto, que o fato de estar em diálise não alterou o hábito alimentar em relação à quantidade de proteínas ingerida por essa população. Porém, observamos que quase a metade dos pacientes estudados (47\%) tinha um consumo protéico abaixo do recomendado de I, $2 \mathrm{~g} / \mathrm{kg} / \mathrm{dia}$. O consumo de proteínas tem sido reportado como baixo em 
pacientes hemodialisados. Inúmeros estudos têm descrito uma média de 0,94 a I,0g proteína/kg/dia. Em nosso meio, Cuppari et al. ${ }^{2}$ também encontraram consumo médio de proteínas de I,03 $\pm \mathrm{I}, 43 \mathrm{~g} / \mathrm{kg} /$ dia, entretanto em cinco dos 10 centros de hemodiálise estudados, o consumo protéico foi menor que $\mathrm{I}, 0 \mathrm{~g} / \mathrm{kg} / \mathrm{dia}$.

Acompanhando o déficit do consumo de proteínas e, principalmente, de energia, observamos reduzido consumo médio de cálcio em nossa população. Em estudos nacionais ${ }^{17,27}$, o consumo de cálcio de pessoas saudáveis, em cidades das Regiões Sul e Sudeste, variava de 974 a $1.182 \mathrm{mg} / \mathrm{dia}$, e em Belém do Pará é de $792 \mathrm{mg} / \mathrm{dia}$, todos bem acima do consumo de cálcio dos pacientes estudados, $307 \mathrm{mg} /$ dia. A orientação dada pelos médicos e nutricionistas para diminuir o consumo de alimentos ricos em fósforo, como o leite e derivados, pode contribuir para esse reduzido consumo de cálcio. Vale ressaltar, porém, que $88 \%$ dos pacientes recebiam suplementação oral de cálcio.

Contrapondo-se ao esperado para uma região farta em pescados, em nosso estudo observamos maior consumo de carne bovina e frango. $O$ difícil acesso a essa fonte protéica na área urbana de Manaus, e o fato de ser uma carne, representada pelo peixe, mais cara que a bovina e a de frango, não favorecem seu consumo, principalmente em nossa população, constituída em geral de pacientes com baixa renda familiar. Vale ressaltar que o consumo de peixe por $48 \%$ dos pacientes é significativamente mais alto, se comparado ao consumo de $7 \%$ nos pacientes em CAPD e 17\% nos pacientes em hemodiálise, em estudo de hábitos alimentares realizados em uma unidade de diálise de São Paulo ${ }^{28}$. O consumo de hortaliças por apenas $42,4 \%$ dos pacientes correspondeu ao esperado, pois o hábito alimentar da região é fortemente influenciado pela cultura indígena que não utiliza esse tipo de alimento na sua rotina alimentar. Ao contrário, o consumo de produtos derivados da mandioca, principalmente a farinha, esteve presente na alimentação de $86,7 \%$ dos pacientes. Entre as frutas consumidas, encontramos a banana pacovã, tucumã, açaí, pupunha, biribá e ingá, espécies exclusivas e abundantes na Região Norte.

A análise conjunta desses dados permite afirmar que a desnutrição é freqüente em pacientes da Região Norte submetidos à hemodiálise, semelhante ao que se observa em outras regiões do país e do mundo. E o reduzido consumo de energia e proteína deve ter implicação na gênese da desnutrição nesse contingente populacional.

\section{SUMMARY}

NUtRITIONAL CONDITION IN CHRONIC RENAL FAILURE PATIENTS TREATED BY HEMODIALYSIS IN AMAZONAS

BACKGROUND. Chronic renal failure patients frequently show malnourishment.

OBJETIVE. The food intake and the nutritional status of 165 chronic renal failure patients on maintenance hemodialysis were studied.

Methods. Nutritional status of 165 patients were studied by anthropometry, biochemical measurements, protein nitrogen appearance rate (PNA) and a average food intake.

RESULTS. The results show that in mean the percent standard body weight, the body mass index (BMI), the mid arm muscle circumference (MAMC) and the triceps skinfold thickness (TSF) were adequate, however, $38 \%$ of women and $27 \%$ of men had the TSF bellow the $5^{\text {th }}$ percentile; $39 \%$ of men and $2 \%$ of women presented MAMC bellow the $5^{\text {th }}$ percent, showing that lose body fat in both sexs and the muscle stores in men. Energy intake was $29.5 \pm 10.2 \mathrm{Kcal} / \mathrm{kg} /$ day and $74 \%$ of the patients consumed less than $35 \mathrm{Kcal} / \mathrm{kg} / \mathrm{day}$, the recommended level for this population. Although the protein intake was in the recommended range $(1.32 \pm 0.4 \mathrm{~g} / \mathrm{kg} /$ day $)$, $47 \%$ of the patients consumed less than $1.2 \mathrm{~g} /$ $\mathrm{Kg} /$ day. Mean serum albumin was $3.96 \pm 0.36$ $\mathrm{g} / \mathrm{dL}$ (normal value). According to TSF and of MAMC criteria (the reference was the $5^{\text {th }}$ percentile), 74 (44.8\%) patients were classified as malnourished.

CONCLUSIONS. The frequency of malnutrition is high in our population and the poor food intake may contribute to this condition. [Rev Assoc Med Bras 2003; 49(I): 72-8]

KEYWORDS: Hemodialysis. Nutritional assessment. Chronic renal failure.

\section{REFERÊNCIAS}

I. Bergstrom J, Lindhom B. Nutrition and adequacy of dialysis. How do hemodialysis and CAPD compare? Kidney Int 1993; 43:539-50.
2. Cuppari L, Draibe AS. Avaliação nutricional de pacientes renais crônicos em programa de hemodiálise. Estudo multicêntrico. Rev Assoc Med Bras 1989; 35:9-14.

3. Acchiardo SR, Moore LW. Malnutrition as the main factor in morbidity and mortality of hemodialysis patients. Kidney Int 1983; 24:S199-S203.

4. ShapiroJl, Argy WP. The unsuitability of bun as a criterion for prescription dialysis. ASAIO Trans 1983; 29: I 29-34.

5. Lowrie EG, Lew NL. Race and diabetes as death risk predictors in hemodialysis patients. Kidney Int 1992; 42:522-31.

6. Khaja RA. Nutrition in maintenance hemodialysis patients in nutritional management of renal disease. Edited: Kopple JD, Massry SG. Baltimore: Williams \& Wilkins; 1997. p.563.

7. Dwyer JT, Kopple JD. Dietary intake and nutritional status in the hemo pilot study population (abstract). JAm Soc Nephrol 1995; 6:576.

8. Blumenkrantz MJ, Kopple JD, Gutman RA, Chan YK, Barbour GL, Roberts C, et al. Methods for assessing nutritional status of patients with renal failure. Am J Clin. Nutr 1980; 33: I567-85.

9. Bergstrom J. Why are dialysis patients malnourished? Am J Kidney Dis 1995; 26:229-4I.

10. Anção MS, Cuppari L. Sistema de apoio à decisão em nutrição-versão2.5. Centro de Informática em Saúde da Universidade Federal de São Paulo. São Paulo: EPM; 1993.

II. Grant JP, Custer PB, Thurlow J. Current techniques of nutricional assessment. Surg Clin North Am 1981; 61:463-73.

12. Frisancho AR. New norms of upper limb fat and muscle areas for assessment of nutritional status. Am J Clin Nutr I 98I ; 34:2540-5.

13. Durnin JYGA, Wormersley]. Body fat assessed from total body density and its estimation from skinfold thickness measurements on $48 \mathrm{I}$ men and women aged from 16 to 72 years. $\mathrm{Br}$ J Nutr 1974; 32:77-97.

14. Bray GA. Definition, measurement and classification of the syndromes of obesity. Int J Obesity 1978; 2:99-II2.

15. Sargent JA, Gotch AS. Mass balance: a quantitative guide to clinical nutritional therapy. J Am Diet Assoc 1979; 75:547-55.

16. Daugirdas JT. Second generation logarithmic estimates of single pool variable volume $\mathrm{Kt} / \mathrm{V}$ : an analysis of error. J Am Soc Nephrol 1993; 4: I205-I 3.

17. Estudo multicêntrico sobre consumo alimentar Revista do Núcleo de Estudo e Pesquisa em Alimentação. NEFA/UNICAMP; 1997.

18. Movilli E, Mombelloni S, Ggiotti M, Maiorcar. Effect of age on protein catabolic rate, morbidity and mortality in uraemic patients with adequate dialysis. Nephrol Dial Transplant 1993; 8:735-9.

19. Chertow GM, Johansen KL, Lew N, Lazarus JM, Lowrie EG. Vintage, nutritional status, and survival in hemodialysis patients. Kidney Int 2000; 57: I |76-8I. 
20. Gotch FA, SargentJA. A mechanistic analysis of the National Cooperative Dialysis Study (NCDS). Kidney Int 1985; 28:526-34.

21 . Lowrie EG, Lew NL. Death risk in hemodialysis patients: The predictive value of commonly measured variables and an evaluation of death rate differences between facilities. Am J Kidney Dis 1990; 15:458-82.

22. Clinical practices guidelines for nutrition in chronic renal failure. K/DOQI. National Kidney Foundation. Am J Kidney. Dis 2000; 35(6 suppl 2):SI-I 40.

23. Blackburn GL, Thornton PA. Nutritional assessment of the hospitalized patient. Med Clin North Am 1979; 63:I I03-I5.
24. Thunberg BJ, Swany AP, Cestero RVM. Crosssectional and longitudinal nutritional measurements in maintenance hemodialysis patients. Am J Clin Nutr I 98I; 34:2005-I 2.

25. Marcen R, Teruel JL, De La Cal MA, Gamez C. The impact of malnutrition in morbidity and mortality in stable hemodialysis patients. Spanish Cooperative Study of Nutrition in Hemodialysis. Nephrol Dial Transplant 1997; |2:2324-3|.

26. Antoniov LD, Shalhoub RJ. Zinc-inducia enhancement of lymphoyte Function and Viability in chronic. Nephron 1985; 40: 13.

27. Universidade Federal do Pará. Departamento de Nutrição. Centro de Referência em
Alimentação e Nutrição. Inquérito de consumo alimentar familiar. Revista do Centro de Referência De Alimentação e Nutrição da Região Norte 1997; I: 10-50.

28. Aveasni CN, Trindade LRT, Draibe AS, Cuppari L. Hábitos alimentares de pacientes em diálise: Comparação entre diálise peritonial ambulatorial continua e hemodiálise. Nutrire 200I; $21: 17-30$.

Artigo recebido: 25/03/2002

Aceito para publicação: 01/10/2002

\section{IMAGEM EM MEDICINA}

Participe da nova seção da Ramb. É um espaço aberto ao leitor, que poderá enviar material de interesse educativo como fotos, ilustrações e exames, acrescido de três linhas explicativas contendo ainda nome do autor e serviço onde foi realizado.

O material poderá ser enviado para a

Rua São Carlos do Pinhal, 324 - Cep 01333-903 S. Paulo - SP - E-mail: ramb@amb.org.br 